\title{
Measurement of Refractive Index of Solid Medium by Critical Angle Method When Air Gap is Present
}

\author{
Hwan Hong Lim, Moon Soo Kwon, Hee Joo Choi, Byoung-Joo Kim, and Myoungsik Cha* \\ Department of Physics, Pusan National University, Busan 609-735, Korea
}

(Received August 19, 2008 : revised September 10, 2008 : accepted September 11, 2008)

\begin{abstract}
A critical angle method was used to measure the index of refraction of a solid medium when an air gap between the prism and the medium is present. The gap effect was analyzed both numerically and experimentally. Since the total internal reflection is severely disturbed by the large gap, determination of the critical angle and the resulting refractive index becomes ambiguous and inaccurate. By using an index matching fluid, we could determine the index of refraction with an uncertainty of $\pm 2 \times 1^{-3}$ even when the gap is as large as $1 \mu \mathrm{m}$.
\end{abstract}

Keywords: critical angle method, refractive index, total internal reflection

OCIS codes : (120.4530) Optical constants; (260.6970) Total internal reflection

\section{INTRODUCTION}

Refractive index is a fundamental physical quantity in characterization or application of an optical material. In optical interactions such as pulse propagation in optical fibers or nonlinear parametric generations, the refractive index of materials is essential for estimating the group-velocity-dispersion and the phase matching conditions [1-3]. Several methods have been used to measure the refractive indices of various optical materials. For transparent bulk materials, the minimum deviation method [4] and interferometric method [5-7] are commonly employed. These methods are by far the most accurate, but restricted to the high quality prism-shaped or plane-parallel samples. For absorbing materials, the ellipsometric method [8] and the prism coupling method $[9,10]$ can be applied to obtain the optical constants near resonances.

In particular, the principle of the critical angle method has long been applied in Abbé refractometers [11]. Since with this method one observes total internal reflection (TIR), and converts the measured critical angle directly to the index value, it has advantages over the other methods. The advantages include easy experimental implementation, no need for complicated fitting, robustness against vibration, and sensitivity to local index near

*Corresponding author: mcha@pusan.ac.kr the surface only. This method can be applied to thick films, bulk materials and absorbing or scattering materials such as biological tissues $[12,13]$. In many practical cases, however, a small air gap is unavoidable between the high-index reference prism and the test sample, which results in an erroneous determination of the critical angle, limiting practical applications of this method. Applying a high pressure to make a better contact between the sample and the prism can result in deformation or damage of the sample surface if it has a soft surface as in organic solids.

In this work, we studied the air gap effect both numerically and experimentally. We filled the gap with a high-index fluid in an attempt to alleviate or remove the gap effect. As a result, we could use the critical angle method for an air gap of several wavelengths, and determine the index of refraction with the same accuracy, as there was little gap.

\section{THEORY}

The experimental setup and the coordinate system are schematically shown in Fig. 1. A beam of light from air is incident with an angle of incidence $\theta_{i}$, on one side of the prism with a well-defined refractive index $n_{1}$ and a base angle $\theta_{p}$. The refracted beam in the prism propagates to the interface between the prism and the air 


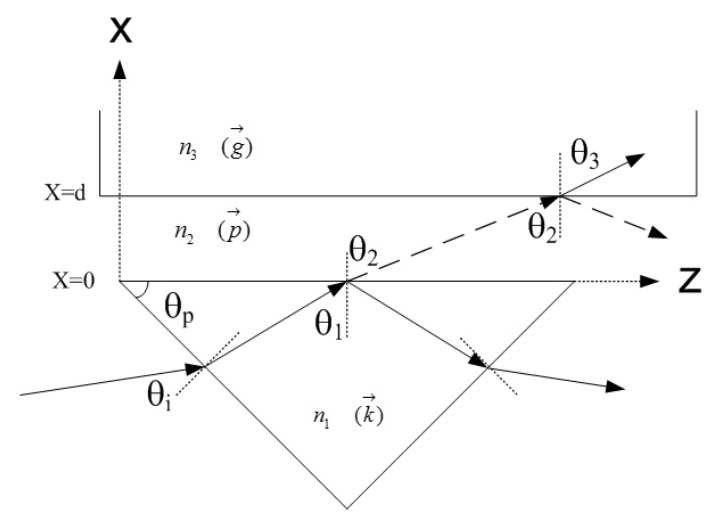

FIG. 1. Schematic diagram of experimental configuration and coordinates. $k, p, g$ are the wavevectors of prism, gap, and test sample, respectively. $n_{1}, n_{2}, n_{3}$ are the refractive indices of the respective media.

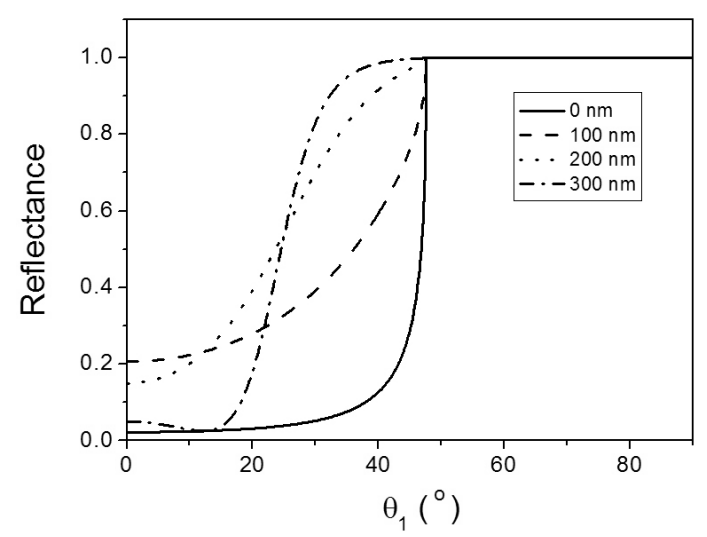

FIG. 2. Reflectance for TE polarization as a function of internal angle of incidence $\theta_{1}$ in the prism with different gaps for $n_{1}=1.977, n_{2}=1.001, n_{3}=1.460$, and $\lambda$ $=543.5 \mathrm{~nm}$.

of refractive index of $n_{2}\left(n_{1}>n_{2}\right)$, and is again reflected and transmitted at the interface between the air and the test sample of refractive index $n_{3}$. The angle of incidence $\theta_{1}$ inside the prism is given by

$$
\theta_{1}=\theta_{p} \pm \sin ^{-1}\left(\frac{n_{a} \sin \theta_{i}}{n_{1}}\right)
$$

where the positive (negative) sign indicates the clockwise (counterclockwise) rotation $\theta_{i}$ of the prism from the normal incidence and $n_{a}$ is the refractive index of the surrounding medium (air in the present experiment). When the prism makes a perfect contact with the sample, we can neglect the small air gap, TIR occurs at the interface when the angle $\theta_{1}$ become larger than the critical angle given by $\theta_{c}=\sin ^{-1}\left(n_{1} / n_{3}\right)$. Hence, if we measure the critical angle, we can directly determine the index of the sample. However, the accuracy of the index measurement is limited by the accuracy in the critical angle, and in practice one cannot make a perfect contact between the sample surface and the reference prism. Ambiguity arises in the determination of the critical angle unless the sample surface is brought to the range of the evanescent field of the prism. Onodera et al. have studied the effects of very small air gap numerically [14]. Here, we systematically carry out the calculation, for the study of air gap larger than the wavelength.

The reflectance of the three-layer system, given by the geometry given in Fig. 1, can be obtained by solving three layers boundary value problem [15]. In the case of TE-polarization, the reflection coefficient $r_{s}$ at the prism interface is given by

$$
r_{s}=\frac{(\alpha+1)(1-\beta)+(\alpha-1)(1+\beta) e^{i 2 p_{x} d}}{(\alpha+1)(1+\beta)+(\alpha-1)(1-\beta) e^{i 2 p_{x} d}}
$$

with

$$
\begin{aligned}
& \alpha=\frac{p_{x}}{g_{x}}, \quad \beta=\frac{p_{x}}{k_{x}} \\
& k_{z}=k_{0} n_{1} \sin \theta_{1} \\
& k_{x}=k_{0} n_{1} \cos \theta_{1} \\
& p_{x}=\left(k_{0}^{2} n_{2}^{2}-k_{z}^{2}\right)^{1 / 2} \\
& g_{x}=\left(k_{0}^{2} n_{3}^{2}-k_{z}^{2}\right)^{1 / 2}
\end{aligned}
$$

where $k_{0}(=2 \pi / \lambda)$ is the wave number in vacuum, $k_{i}$ $\left(p_{i}, g_{i}\right)$ is the $\mathrm{i}$-th cartesian component of the wavevector in medium $1(2,3)$, and $d$ represents the gap size. Here, all of the media are assumed to be non-magnetic, $\mu=$ $\mu_{0}$, and we also neglect the reflections at the entrance and exit planes of the prism.

When the incident light is TM-polarized, the reflection coefficient $r_{p}$ is given by

$$
r_{p}=\frac{(1+N)(1-M)-(1-N)(1+M) e^{i 2 p_{x} d}}{(1+N)(1+M)-(1-N)(1-M) e^{i 2 p_{x} d}}
$$

with

$$
N=\left(\frac{n_{3}}{n_{2}}\right)^{2} \alpha, \quad M=\left(\frac{n_{1}}{n_{2}}\right)^{2} \beta
$$

The system reflectance $\left(\left|r_{s, p}\right|^{2}\right)$ in Eqs. (2) and (8) are plotted as a function of the internal angle of incidence in the prism $\theta_{1}$ to study the air gap effects. The results for TE and TM polarizations are shown in Figs. 2 and 3, 


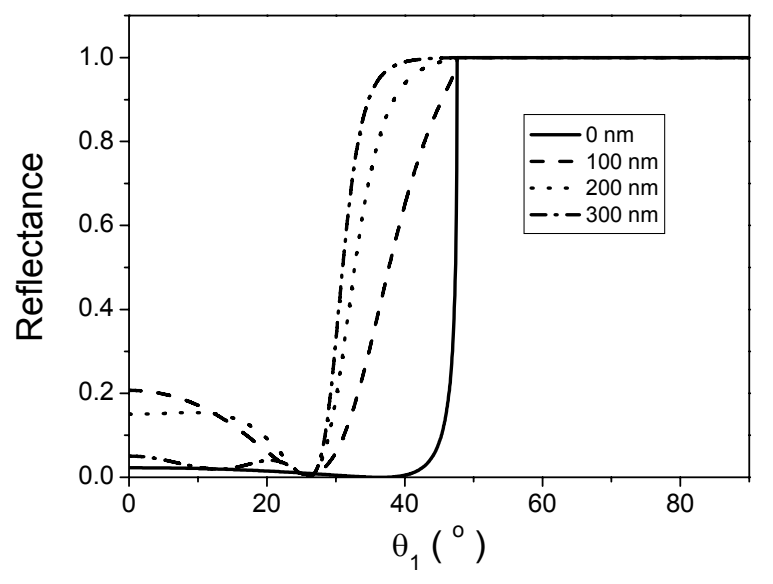

FIG. 3. Reflectance for TM polarization with the same conditions in Fig. 2.

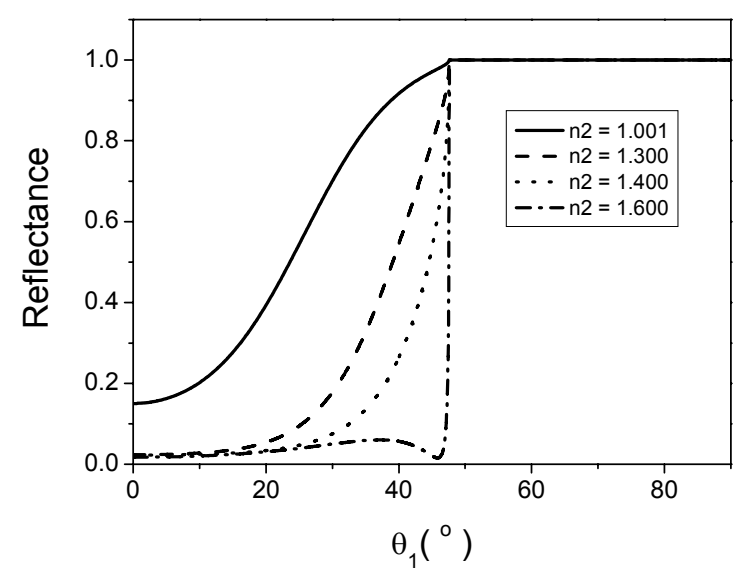

FIG. 4. Reflectance for TE polarization when gap is 200 $\mathrm{nm}$, for different refractive indices of the gap material.

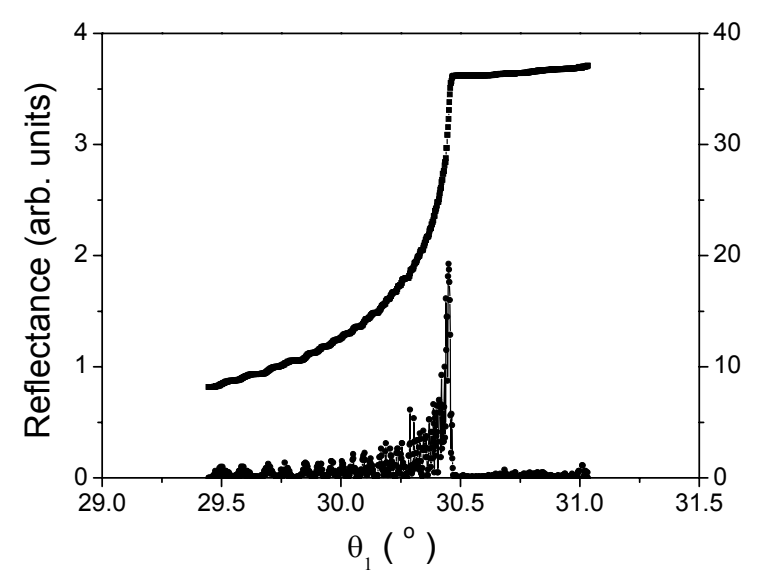

FIG. 5. Measured reflectance of air (top) and its derivative (bottom) for experimental determination of the critical angle

respectively, with the parameters $n_{1}=1.977, n_{2}=1.001$, $n_{3}=1.460$, and $\lambda=543.5 \mathrm{~nm}$. When the gap increases, the sharp variation of the reflectance around the critical angle is blunted in both cases. It results in an ambiguous determination of the critical angle.

Use of an index matching fluid is a simple and powerful method for reducing the gap effect. Figure 4 shows the reflectance curve for several values of the index $n_{2}$ of the gap material. If the gap is filled with an index-matching fluid, we can see that the gap effect is reduced drastically as compared to Fig. 2. Especially, an index matching fluid with the refractive index between those of the prism and the sample can perfectly remove the gap effect.

\section{EXPERIMENTS}

A He-Ne laser $(\lambda=543.5 \mathrm{~nm}$, Melles Griot) was used as a light source. The natural beam from the laser ( $1 / \mathrm{e}^{2}$-radius at the waist: $\left.0.24 \mathrm{~mm}\right)$ was used in the experiment. The polarization direction of the laser was fixed to TE-polarization. A gadolinium gallium garnet (GGG) prism was used as a high-index reference whose refractive index is known to be 1.977 at $543.5 \mathrm{~nm}$. The test sample was a fused silica plate (Esco Products) because its dispersion is well-known [16, 17].

The prism and the sample were mounted on a computercontrolled stepping motor with steps of $0.004^{\circ}$. The accuracy of the steps was assured by checking the retroreflection of the laser beam from the prism surface after $360^{\circ}$ rotation. The prism base angle $\left(\theta_{p}\right)$ was measured to be $44.976 \pm 0.004^{\circ}$. The power of reflected beam from interface between the prism base and test sample was measured around the critical angle with a silicon photodiode with a wide aperture. In order to study the gap effect experimentally, we controlled the gap size with $1 \mu \mathrm{m}$ diameter polystyrene spheres as a spacer between the GGG prism and the fused silica plate. An index matching fluid with index of 1.50 (Norland Products) was used to fill the gap.

\section{RESULTS AND DICUSSION}

In order to verify the validity of our system, we first measured the refractive index of air at room temperature. The prism was rotated without the sample, resulting in a reflectance shown in Fig. 5. The measured reflected power varied sharply around the critical angle of the prism-air interface. Also shown in the figure is the derivative of the measured data with respect to the internal angle of incidence $\theta_{1}$. The derivative is obtained by numerically calculating the difference between the adjacent measured data. The angle at the maximum derivative is $30.450^{\circ}$. We identified this angle as the critical angle for the TIR from prism to air. From this value, we determined the refraction index value of air to be 1.002. Since the index of air is known to be 1.00026 [18], we estimate the uncertainty of our measurement 


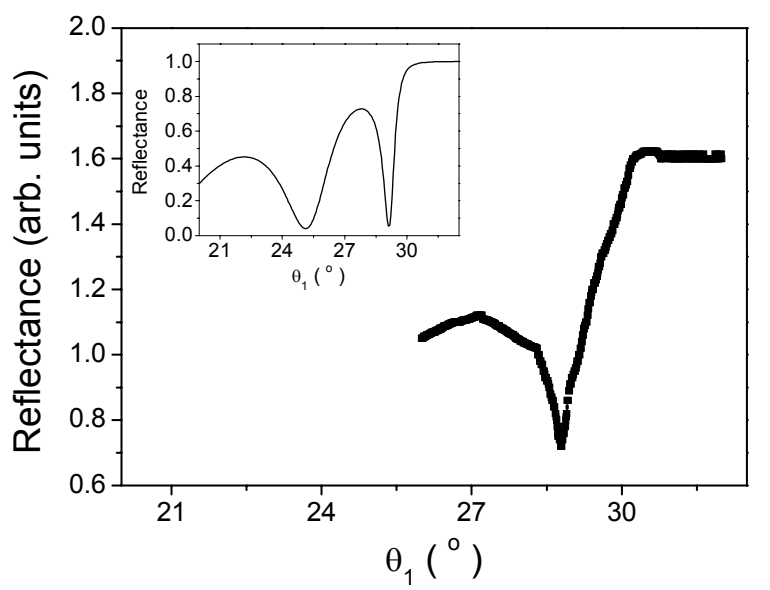

FIG. 6. Reflectance for TM polarization with the same conditions in Fig. 2.

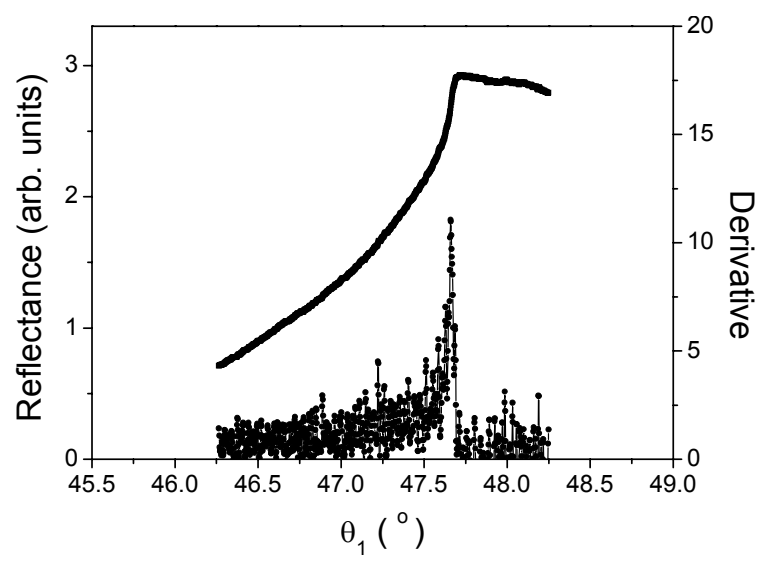

FIG. 7. Reflectance for TE polarization when gap is 200 $\mathrm{nm}$, for different refractive indices of the gap material.

to be 0.002 . A possible cause for this discrepancy is the Gaussian beam effect [14]. For a narrow Gaussian beam, the wavevector is not unique as opposed to the ideal plane wave due to self-diffraction, which makes the reflectance change blunt around the critical angle. The Gaussian beam effect could be alleviated if a larger collimated beam is used. The second cause would be inaccurate definition of the angles such as prism base angle and normal incidence. In this case, the absolute value of the critical angle or the refractive index cannot be determined with accuracy, but relative measurements could be still valid. Thus, the measurement accuracy can be improved by using a well-calibrated reference sample [19]. Taking the angle at the maximum derivative to be the critical angle could also lead to an additional error, when the reflectance change at the critical angle is not ideally sharp.

Figure 6 shows the measured reflected power for a fused silica plate when the air gap was kept $1 \mu \mathrm{m}$ by the polystyrene spacer. The interference effect dominates the TIR (oscillation in the reflectance), making the determination of critical angle extremely ambiguous and inaccurate. In order to alleviate the air gap effect, we filled the gap with index-matching fluid $\left(n_{2}=1.50\right)$, and measured the reflected power as shown in Fig. 7. Since the refractive index of the fluid is larger than that of the sample, the critical angle could be clearly defined as the angle of the maximum derivative in spite of the large gap. From this, the index of the fused silica plate was determined to be $1.461 \pm 0.002$, which agrees well with the value calculated by the Sellmeier's formula [16] within the error of our measurement system.

\section{CONCLUSION}

We used the critical angle method to measure the refractive index of a solid medium. The effect of the gap between the prism and test sample were analyzed both numerically and experimentally. The total internal reflection was disturbed by the large gap, so that the determination of critical angle became extremely ambiguous and inaccurate. Although the large air gap was present, we could determine the index of refraction of the samples with an uncertainty of $\sim 10^{-3}$, by filling the gap with an index-matching fluid. Our method can be use to measure the index of refraction of soft materials such as organic solids.

\section{ACKNOWLEDGMENT}

This work was supported by the Korea Research Foundation Grant \# KRF-2005-202-C00151, and by the Ministry of Knowledge and Economy of Korea through the Ultrashort Quantum Beam Facility Program.

\section{REFERENCES}

[1] G. P. Agrawal, Nonlinear fiber optics (Academic Press, San Diego 1989), Ch. 3.

[2] R. W. Boyd, Nonlinear optics (Academic Press, San Diego 1992), Ch. 2.

[3] N. E. Yu, Y. Lee, C. Jung, and J. Lee, "Efficient singlepass optical parametric generation and amplification using a periodically poled stoichiometric lithium tantalate," J. Opt. Soc. Korea, vol. 11, no 4, pp. 192-195, 2007.

[4] W. L. Bond, "Measurement of the refractive indices of several crystals," J. Appl. Phys., vol. 36, pp. 1674-1677, 1965 .

[5] M. S. Shumate, "Interferometric Measurement of Large Indices of Refraction,” Appl. Opt., vol. 5, no.2, pp. 327331, 1966.

[6] J. F. H. Nicholls, B. Henderson, and B. H. T. Chai, "Accurate determination of the indices of refraction of nonlinear optical crystals," Appl. Opt. vol. 36, no. 33, pp. 8587-8594, 1997.

[7] U. Schlarb and K. Betzler, "Interferometric measurement 
of refractive indices of $\mathrm{LiNbO}_{3}$, Ferroelectrics, vol. 126, no. 1, pp. 39-44, 1992.

[8] T. Yamaguchi and H. Takahashi, "Ellipsometric method for separate measurements of $\mathrm{n}$ and $\mathrm{d}$ of a transparent film," Appl. Opt., vol. 14, no. 8, pp. 2010-2015, 1975

[9] A. C. Adams, D. P. Schinke, and C. D. Capio, "An evaluation of the prism coupler for measuring the thickness and refractive index of dielectric films on silicon substrates," J. Electrochem. Soc., vol. 126, pp. 1539-1543, 1979.

[10] R. Ulrich and R. Torge, "Measurement of Thin Film Parameters with a Prism Coupler,” Appl. Opt., vol. 12, no. 12, pp. 2901-2908, 1973

[11] www.atago.net

[12] H. Li and S. Xie, "Measurement method of the refractive index of biotissue by total internal reflection," Appl. Opt., vol. 35, no.10, pp. 1793-1795, 1996

[13] Q. W. Song, C. Y. Ku, C. Zhang, R. B. Gross, R. R.
Birge, and R. Michalak, "Modified critical angle method for measuring the refractive index of bio-optical materials and its application to bacteriorhodopsin," J. Opt. Soc. Am. B., vol. 12, no. 5, pp. 797-803, 1995

[14] H. Onodera, I. Awai, and J. Ikenoue, "Refractive-index measurement of bulk materials: prism coupling method," Appl. Opt., vol. 22, no. 8, pp. 1194-1197, 1983

[15] J. D. Jackson, Classical Electrodynamics, $3^{\text {rd }}$ ed. (John Wiley and Sons, New York 1999), Ch. 7.

[16] I. H. Malitson, "Interspecimen comparison of the refractive index of fused silica," J. Opt. Soc. Am., vol. 55, pp. 1205-1209, 1965

[17] J. H. Wray and J. T. Neu, "Refrective index of several glasses as a. function of wavelength and temperature," J. Opt. Soc. Am., vol. 59, pp. 774-776, 1969

[18] B. Edlen, "The refractive index of air," Metrologia, vol. 2, pp. 71-80, 1966

[19] www.nist.gov 